\title{
Outcomes after neoadjuvant or adjuvant chemotherapy for cT2-4N0-1 non-small cell lung cancer: A propensity-matched analysis
}

Whitney S. Brandt, MD, ${ }^{a}$ Wanpu Yan, MD, ${ }^{a}$ Jian Zhou, MD, ${ }^{a}$ Kay See Tan, PhD, ${ }^{b}$ Joseph Montecalvo, MD, ${ }^{c}$ Bernard J. Park, MD, ${ }^{\mathrm{a}}$ Prasad S. Adusumilli, MD, ${ }^{\mathrm{a}}$ James Huang, MD, ${ }^{\mathrm{a}}$ Matthew J. Bott, MD, ${ }^{\mathrm{a}}$ Valerie W. Rusch, MD, ${ }^{a}$ Daniela Molena, MD, ${ }^{\mathrm{a}}$ William D. Travis, MD, ${ }^{\mathrm{c}}$ Mark G. Kris, MD, ${ }^{\mathrm{d}}$ Jamie E. Chaft, MD, ${ }^{\mathrm{d}}$ and David R. Jones, $\mathrm{MD}^{\mathrm{a}}$

\section{ABSTRACT}

Objective: Comparative survival between neoadjuvant chemotherapy and adjuvant chemotherapy for patients with cT2-4N0-1M0 non-small cell lung cancer has not been extensively studied.

Methods: Patients with cT2-4N0-1M0 non-small cell lung cancer who received platinum-based chemotherapy were retrospectively identified. Exclusion criteria included stage IV disease, induction radiotherapy, and targeted therapy. The primary end point was disease-free survival. Secondary end points were overall survival, chemotherapy tolerance, and ability of Response Evaluation Criteria In Solid Tumors response to predict survival. Survival was estimated using the Kaplan-Meier method, compared using the log-rank test and Cox proportional hazards models, and stratified using matched pairs after propensity score matching.

Results: In total, 330 patients met the inclusion criteria $(n=92 /$ group after propensity-score matching; median follow-up, 42 months). Five-year diseasefree survival was $49 \%$ (95\% confidence interval, 39-61) for neoadjuvant chemotherapy versus $48 \%$ (95\% confidence interval, $38-61$ ) for adjuvant chemotherapy $(P=.70)$. On multivariable analysis, disease-free survival was not associated with neoadjuvant chemotherapy or adjuvant chemotherapy (hazard ratio, 1.1; 95\% confidence interval, $0.64-1.90 ; P=.737$ ), nor was overall survival (hazard ratio, $1.21 ; 95 \%$ confidence interval, $0.63-2.30 ; P=.572$ ). The neoadjuvant chemotherapy group was more likely to receive full doses and cycles of chemotherapy $(P=.014 / 0.005)$ and had fewer grade 3 or greater toxicities $(P=.001)$. Response Evaluation Criteria In Solid Tumors response to neoadjuvant chemotherapy was associated with disease-free survival $(P=.035) ; 15 \%$ of patients receiving neoadjuvant chemotherapy (14/92) had a major pathologic response.

Conclusions: Timing of chemotherapy, before or after surgery, is not associated with an improvement in overall or disease-free survival among patients with cT2-4N0-1M0 non-small cell lung cancer who undergo complete surgical resection. (J Thorac Cardiovasc Surg 2019;157:743-53)

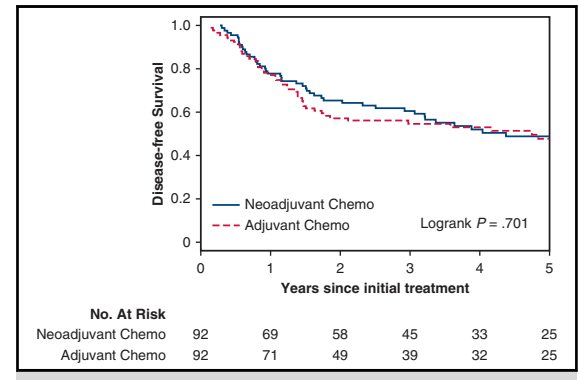

Five-year DFS for the NC versus AC group.

\section{Central Message}

Timing of chemotherapy (before or after surgical resection) is not associated with survival for patients with cT1-3N0-1M0 NSCLC; however, tolerance is improved with neoadjuvant therapy.

\section{Perspective}

For patients with cT1-3N0-1M0 NSCLC, timing of chemotherapy is not associated with survival. The benefits of $\mathrm{NC}$ over AC, including improved tolerance, ability to stop/alter therapy if no response, and potential to determine prognosis based on tumor response, make $\mathrm{NC}$ a favorable option for patients and clinicians.

See Editorial page 754.

See Editorial Commentary page 756.

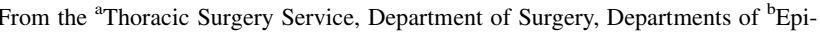
demiology and Biostatistics and ${ }^{\mathrm{c} P a t h o l o g y, ~ a n d ~}{ }^{\mathrm{d}}$ Thoracic Oncology Service, Department of Medicine, Memorial Sloan Kettering Cancer Center, New York, NY. This work was supported by National Institutes of Health Grants R01 CA217169 (to D.R.J.) and T32 CA009501 (to W.S.B.). This work was also supported, in part, by the National Institutes of Health/National Cancer Institute Cancer Center Support Grant P30 CA008748. The sponsor played no role in study design; in the collection, analysis, and interpretation of data; in the writing of the report; or in the decision to submit the article for publication.

Institutional Review Board Approval: \#16-1395, approved September 26, 2016.
}

W.S.B. and W.Y. contributed equally to this work.

Received for publication June 4, 2018; revisions received Aug 31, 2018; accepted for publication Sept 18, 2018; available ahead of print Nov 8, 2018.

Address for reprints: David R. Jones, MD, Thoracic Surgery Service, Memorial Sloan Kettering Cancer Center, 1275 York Ave, Box 7, New York, NY 10065 (E-mail jonesd2@mskcc.org).

$0022-5223 / \$ 36.00$

Copyright $(2018$ by The American Association for Thoracic Surgery https://doi.org/10.1016/j.jtcvs.2018.09.098 


$$
\begin{aligned}
& \text { Abbreviations and Acronyms } \\
& \text { AC } \quad=\text { adjuvant chemotherapy } \\
& \text { ASMD }=\text { absolute standardized mean difference } \\
& \text { CI } \quad=\text { confidence interval } \\
& \text { CT } \quad \text { computed tomography } \\
& \text { DFS }=\text { disease-free survival } \\
& \text { HR } \quad=\text { hazard ratio } \\
& \text { MPR }=\text { major pathologic response } \\
& \text { NC }=\text { neoadjuvant chemotherapy } \\
& \text { NCCN }=\text { National Comprehensive Cancer } \\
& \text { Network } \\
& \text { NSCLC } \text { non-small cell lung cancer } \\
& \text { OS }=\text { overall survival } \\
& \text { PET }=\text { positron emission tomography } \\
& \text { RECIST }=\text { Response Evaluation Criteria In Solid } \\
& \text { Tumors } \\
& \text { SUV }=\text { standardized uptake value }
\end{aligned}
$$

(U) Scanning this QR code will
take you to the article title
page to access supplementary
information.

We have recently shown that even with a complete resection (R0) in patients with pathologic node-negative lung adenocarcinoma, there was a high incidence $(20 \%-50 \%)$ of distant recurrence for increasing T-stage tumors, suggesting that surgery alone in this patient population is inadequate therapy. ${ }^{1}$ The National Comprehensive Cancer Network guidelines recommended surgery followed by adjuvant chemotherapy (AC) for patients with T2-4N0-1 non-small cell lung cancer (NSCLC), with a footnote that neoadjuvant chemotherapy (NC) should also be considered for these patients. $^{2}$ The recommendation for AC is based on multiple phase III randomized controlled trials and a meta-analysis that established an approximately $5 \%$ better 5 -year overall survival (OS) for surgery plus AC versus surgery alone..$^{3-5}$ Although associated with improved survival, up to $51 \%$ of patients who receive AC experience adverse events, $23 \%$ of which are grade $4 .{ }^{5}$ In addition, compliance with AC regimens is poor: In one study, $9 \%$ of patients did not receive any of the prescribed $\mathrm{AC}$, only $59 \%$ received the full dose, and $25 \%$ received $\leq 2$ cycles of $\mathrm{AC}^{4}{ }^{4}$

$\mathrm{NC}$ offers potential benefits over AC for patients with cT2-4N0-1 NSCLC, including additional time for preoperative cessation of smoking, reduction in tumor size, treatment of micro-metastatic disease, and ability to assess treatment response, which can affect decisions on appropriate selection of additional adjuvant therapies. Radiographic tumor response to NC may also provide additional prognostic information and allows the ability to consider cessation or switch of therapy in the absence of a response. ${ }^{6}$ In addition, $\mathrm{NC}$ has been associated with better tolerance and compliance, as demonstrated in a phase III randomized trial in which $97 \%$ of patients in the $\mathrm{NC}$ group started planned chemotherapy, compared with $66 \%$ in the AC group.

The preponderance of available data support surgery followed by AC over surgery alone. In contrast, the evidence base supporting the use of NC for patients with cT2-4N01 NSCLC is limited, and comparisons with AC are not robust and have limitations. ${ }^{7}$ With the increasing interest in the use of induction immunotherapy for resectable NSCLC, it is becoming ever more important to understand the contemporary effects of $\mathrm{NC}$ versus $\mathrm{AC}$ on outcomes in these patients. ${ }^{8}$ Therefore, the primary objective of this study was to evaluate disease-free survival (DFS) among patients with cT2-4N0-1 NSCLC treated with NC or AC. Secondary objectives included assessment of OS and compliance with chemotherapy regimen(s). In addition, among patients receiving $\mathrm{NC}$, the relationship between survival and major pathologic response (MPR) rates and radiographic response to chemotherapy were investigated.

\section{MATERIALS AND METHODS Patient Population}

After Institutional Review Board approval (Protocol \# 16-1395), we performed a retrospective review of a prospectively maintained, singleinstitution surgical database for patients with cT2-4N0-1 NSCLC who were treated with platinum-based chemotherapy and surgical resection from January 2000 to December 2015. The decision to administer NC versus proceed directly to surgical resection was based on physician assessment and multidisciplinary tumor board discussion. At our institution, patients who receive $\mathrm{NC}$ are typically reevaluated radiographically after 2 cycles of therapy; if there is no response, the decision is to proceed directly to surgery or to switch chemotherapy agents as previously described by our group. ${ }^{6}$ Patients were excluded from analysis if they had undergone induction radiotherapy, any targeted therapy (with or without chemotherapy), both $\mathrm{NC}$ and $\mathrm{AC}$, or a nonconventional chemotherapy regimen (including additional treatment for a separate metastatic cancer); if they did not undergo a pretreatment positron emission tomography [PET] scan; if they had stage IV disease (American Joint Committee on Cancer 8th edition) or microscopic or macroscopic residual disease (R1/R2 resection); or if more than 90 days had elapsed between surgery and either NC or AC. Patient demographic characteristics, primary tumor maximum standardized uptake value $\left(\mathrm{SUV}_{\max }\right)$, tumor response to $\mathrm{NC}$, chemotherapy details, pathologic tumor subtype (American Joint Committee on Cancer 8th edition), postoperative complications, and follow-up data were documented. Patients who received NC underwent restaging with post-treatment computed tomography (CT) and PET/CT. Mediastinal surgical restaging was performed on a selective basis.

Tumor response to NC was assessed by change in size on CT scan in accordance with Response Evaluation Criteria In Solid Tumors (RECIST). ${ }^{10}$ Pathologic responses to NC were verified by re-review of pathologic slides and was confirmed by a pathologist (J.M., W.T.). MPR was defined as $90 \%$ or greater necrosis of the tumor. ${ }^{11}$ Chemotherapy tolerance was defined as the receipt of full doses and full cycles without 
alteration secondary to intolerance or adverse reactions. Adverse reactions to chemotherapy and postoperative complications were graded according to the Common Terminology Criteria for Adverse Events (versions 5.0 and 4.0, respectively). Prolonged air leak was defined as air leak lasting more than 5 days. ${ }^{12,13}$

Patient follow-up was performed by history and physical examination and CT scan every 6 months for the first 2 years and then annually thereafter, in accordance with National Comprehensive Cancer Network guidelines. The timing and location of recurrences were noted. Metachronous primary lung cancers were differentiated from recurrent disease using the criteria established by Martini and Melamed, ${ }^{14}$ as well as using molecular genomic data, when available.

\section{Statistical Analysis}

To reduce potential selection bias related to a nonrandomized cohort, we included results from propensity score-matched analyses and generated 2 groups (NC and $\mathrm{AC}$ ) with comparable characteristics (ie, balanced). Propensity scores were computed as the conditional probability of receiving $\mathrm{AC}$ (vs NC) using a logistic regression model that included 10 variables: year of surgery (2000-2005, 2006-2010, 2011-2015), age at surgery, gender, body mass index $\left(\mathrm{kg} / \mathrm{m}^{2}\right)$, Charlson comorbidity score, procedure (pneumonectomy vs bilobectomy, lobectomy, or segmentectomy), tumor laterality, histologic subtype (adenocarcinoma, squamous, vs large cell, mixed, or other), clinical stage (IB, IIA, IIB, IIIA), and pretreatment tumor SUV $_{\text {max }}$.

Propensity score matching resulted in matched pairs of patients (1 from each chemotherapy group) with a similar propensity for receiving AC versus NC. Propensity score-matched pairs were identified without replacement using a 1:1 nearest-neighbor matching algorithm with estimated caliper width. The caliper width was based on the recommendation by Austin ${ }^{15,16}$ (equal to 0.2 of the standard deviation of the logit of the propensity score). Balance in variables between groups was assessed by the absolute standardized mean difference (ASMD) before and after the matching procedure. An ASMD less than 0.1 indicates balance in the covariates between $\mathrm{NC}$ and $\mathrm{AC}$. After matching, 92 pairs that were comparable across patient characteristics were available for analysis. Univariable and multivariable analyses were performed on the matched set. As a sensitivity analysis and to model an intention-to-treat analysis, the propensity score-matching procedures were repeated to include a subset of previously excluded patients (R1/R2 resection or stage IV disease). There were too few $\mathrm{R} 1 / \mathrm{R} 2$ and stage IV cases to achieve adequate balance between the 2 chemotherapy groups, because none of these additional cases had an adequate match in the other group. The resulting propensity score-matched cohort, obtained using an optimal caliper width that ensured balanced between the 2 chemotherapy groups (not shown), did not include any R1/R2 or stage IV cases. The study analysis was limited to patients who had $\mathrm{R} 0$ resection.

To assess the effect of $\mathrm{NC}$ and $\mathrm{AC}$ on long-term outcomes, we chose DFS and OS as the end points of interest. Survival was measured from the time of the first treatment to the time of death (OS) or recurrence or death (DFS). Patients who do not die or experience recurrence were censored on the date of the last follow-up. DFS and OS were estimated using the Kaplan-Meier approach and compared between NC and AC groups using the log-rank test. Associations between variables and DFS and OS were quantified using Cox proportional hazard models. All analyses were stratified by matched pairs. Chemotherapy tolerance, adverse reactions to chemotherapy, and postoperative complications were compared between the propensity score-matched NC and AC groups using Fisher exact test.

Last, in the NC group, we evaluated whether RECIST, tumor pathologic response, and ypStage were associated with DFS and OS. For this analysis, we used the entire cohort of patients who underwent NC $(n=142)$. Survival was estimated using the Kaplan-Meier approach and compared between RECIST classifications and between MPR strata $(<90 \%$ vs $\geq 90 \%$ tumor necrosis) using the log-rank test.
Statistical analyses were conducted using R 3.1.1 (R Development Core Team, Vienna, Austria). The propensity score-matching procedures used the Matchlt and tableone packages. All statistical tests were 2-sided.

\section{RESULTS \\ Patient Population}

In total, 330 patients met the inclusion criteria $(\mathrm{NC}=142, \mathrm{AC}=188)$ (see CONSORT diagram, Figure 1). Eighty-eight percent of patients who received NC had an R0 resection (142/162). Patients with incomplete resection had microscopic tumor (R1) present at the margin $(\mathrm{N}=9)$, positive pericardial fluid $(\mathrm{N}=1)$, or bulky tumor/ nodes inseparable from the superior vena cava $(\mathrm{N}=4)$ or aorta $(\mathrm{N}=3)$, or the surgeon did not believe a R0 resection was possible even with a pneumonectomy $(\mathrm{N}=3)$. Of the 20 patients in who had an incomplete resection and $\mathrm{NC}$, 13 died (65\%). As stated in the "Materials and Methods" section, when the propensity score matching was performed including these patients, all were eliminated as part of that analysis. Before propensity score matching, NC patients were more likely to have earlier year of surgery, pneumonectomy, higher clinical stage, higher pretreatment tumor $\mathrm{SUV}_{\max }$, and adenocarcinoma (Table 1). After matching, the ASMD between AC and NC for year of surgery, age, gender, body mass index, Charlson comorbidity score, resection type, tumor histologic subtype, pretreatment tumor SUV $\mathrm{Sax}_{\text {max }}$, and clinical stage were all less than 0.1, indicating proper matching (Table 1). Additional clinicopathologic characteristics of the matched cohort are listed in Table E1.

\section{Survival and Recurrence}

After propensity score matching, both groups ( $\mathrm{NC}$ and AC) comprised 92 patients. The median duration of follow-up was 69.6 months (interquartile range [IQR], 38.8-121.2 months). Median follow-up for the propensity score-matched NC group was 74.4 months (IQR, 42138 months) versus 64.4 months (IQR, 42.7-108 months). Postoperative radiation therapy was administered to $11 \%$ of patients in the $\mathrm{NC}$ group and $4 \%$ of patients in the $\mathrm{AC}$ group (Table E1; Fisher exact test $P=.16$ ). In this propensity score-matched cohort, 33 patients in the NC group had recurrence, 4 of which were locoregional, 20 were distant, and 9 were both distant and locoregional. In the AC group, of 41 patients with recurrence, 1 was locoregional only, 34 were distant recurrences, and 6 were both locoregional and distant.

We first evaluated whether there was an association between DFS and NC or AC. Median DFS was 4.38 years (95\% confidence interval [CI], 2.92-6.90 years) in the $\mathrm{NC}$ group versus 4.74 years ( $95 \% \mathrm{CI}, 1.63$ to not available) in the AC group. The 5-year DFS was $48 \%(95 \%$ CI, 39 $61)$ for the NC group versus $48 \%(95 \% \mathrm{CI}, 38-60)$ for the NC group (Figure 2, $A$; log-rank $P=.701$ ). On 
548 cT2-4NO-1MO NSCLC, Resection, \& Chemotherapy (Jan 2000-Dec 2015)

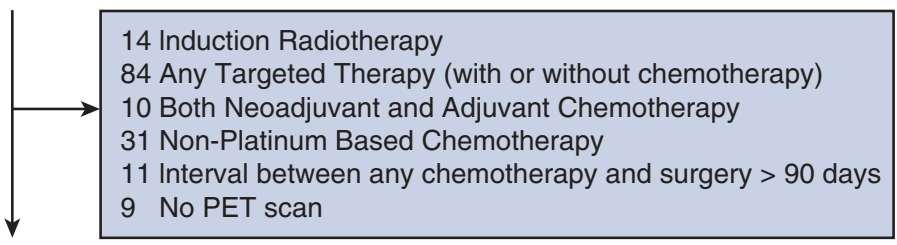

368 Surgery \& Platinum-Based Chemotherapy

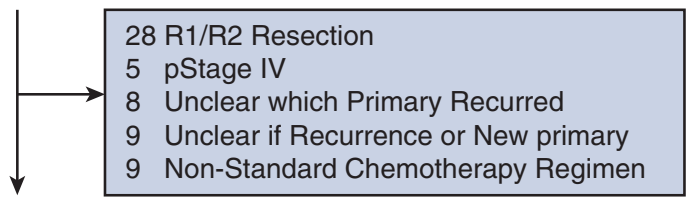

330 cT2-4N0-1M0, pMO NSCLC with R0 Resection

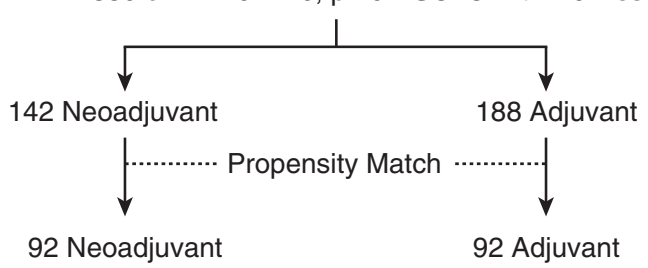

FIGURE 1. CONSORT diagram. NSCLC, Non-small cell lung cancer; PET, positron emission tomography.

univariable analysis, there was no significant association between DFS and NC or AC (AC vs NC hazard ratio [HR], 1.09; 95\% CI, 0.61-1.95; $P=.768$ ). We next evaluated DFS using a multivariable model, and similarly there was no significant association between DFS and NC or AC (AC vs AC HR, 1.10; 95\% CI, 0.64-1.90; $P=.737$ ) (Table 2).

Median OS was 9.22 years ( $95 \%$ CI, 5.14-12.92 years) in the NC group versus 8.98 years $(95 \%$ CI, 4.62 to not available) in the AC group. The 5-year OS was $64 \%(95 \% \mathrm{CI}$, 54-75) for the NC group versus $61 \%(95 \% \mathrm{CI}, 50-74)$ for the AC group (Figure 2, $B$; log-rank $P=.768$ ). On univariable analysis, there was no significant association between OS and NC or AC (HR, 1.09; 95\% CI, 0.61-1.95; $P=.77)$. On multivariable analysis, there remained no significant association between $\mathrm{OS}$ and $\mathrm{NC}$ or $\mathrm{AC}$ (AC vs NC HR, 1.21; 95\% CI, 0.63-2.30; $P=.572$ ) (Table 2).

We also evaluated whether postoperative complications and operative approach differed between $\mathrm{NC}$ and AC. There were $41(45 \%)$ total postoperative complications in the $\mathrm{NC}$ group versus $32(35 \%)$ in the AC group (Fisher exact test $P=.23)$. Most complications were low grade with only 13 patients in the $\mathrm{NC}$ group $(14 \%)$ having grade 3 or greater complications, compared with 6 patients in the $\mathrm{AC}$ group (7\%) (Fisher exact test $P=.14$ ) (Table E2). In the unmatched cohort, 30-day mortality was $0.7 \%$ for the NC group versus $0 \%$ for the $\mathrm{AC}$ group $(P=.4)$; 90 -day mortality was $2.8 \%$ for the $\mathrm{NC}$ group versus $1.1 \%$ for the $\mathrm{AC}$ group $(P=.4)$. After propensity score matching, 30-day mortality was $1.1 \%$ for the NC group versus $0 \%$ for the AC group $(P=.99)$, and 90 -day mortality was $3.3 \%$ for the NC group versus $2.2 \%$ for the $\mathrm{AC}$ group $(P=.99)$. Unsurprisingly, after propensity score matching, fewer patients in the $\mathrm{NC}$ group underwent minimally invasive approach $(7$ patients, 6.9\%) versus the AC group (19 patients, 19\%) $(P=.02)$.

\section{Chemotherapy Tolerance}

Tolerance and completion of chemotherapy were examined using the propensity score-matched cohort. There was no significant association between $\mathrm{NC}$ or $\mathrm{AC}$ and whether patients received cisplatin-based chemotherapy or carboplatin-based chemotherapy $(P=.8$, Appendix Table 1). In the propensity score-matched cohort, 6 patients in the NC group stopped therapy completely versus 20 in the $\mathrm{AC}$ group. In addition, 9 had their regimens altered in the $\mathrm{NC}$ group versus 7 in the $\mathrm{AC}$ group. We found that patients in the $\mathrm{NC}$ group were more likely to receive the full cycle and full dose and had fewer high-grade toxicities related to chemotherapy than patients in the AC group (Table 3).

\section{Response to Neoadjuvant Chemotherapy}

Finally, we examined whether response to $\mathrm{NC}$ provides additional prognostic information about survival. Characteristics of the entire NC cohort $(n=142)$, including 
TABLE 1. Distribution of variables between adjuvant and neoadjuvant groups before and after propensity score matching

\begin{tabular}{|c|c|c|c|c|c|c|}
\hline \multirow[b]{2}{*}{ Variable } & \multicolumn{3}{|c|}{ Before propensity score matching } & \multicolumn{3}{|c|}{ After propensity score matching } \\
\hline & Neoadjuvant $(N=142)$ & Adjuvant $(\mathbf{N}=188)$ & ASMD & $\overline{\text { Neoadjuvant }(\mathbf{N}=92)}$ & Adjuvant $(\mathbf{N}=92)$ & $\overline{\text { ASMD }}$ \\
\hline \multicolumn{7}{|l|}{ Year of surgery } \\
\hline $2000-2005$ & $55(39)$ & $44(23)$ & 0.346 & $25(27)$ & $22(24)$ & 0.081 \\
\hline 2006-2010 & $38(27)$ & $70(37)$ & & $28(30)$ & $28(30)$ & \\
\hline 2011-2015 & $49(35)$ & $74(39)$ & & $39(42)$ & $42(46)$ & \\
\hline Age at surgery, y & $65(58-73)$ & $65(59-72)$ & 0.021 & $64(58-74)$ & $65(59-71)$ & 0.017 \\
\hline \multicolumn{7}{|l|}{ Gender } \\
\hline Female & $75(53)$ & $86(46)$ & 0.142 & $40(43)$ & $39(42)$ & 0.022 \\
\hline Male & $67(47)$ & $102(54)$ & & $52(57)$ & $53(58)$ & \\
\hline BMI, $\mathrm{kg} / \mathrm{m}^{2}$ & $26.4(23.6-29.7)$ & $27.2(24.0-30.4)$ & 0.152 & $26.6(23.8-30.0)$ & $27.3(23.7-29.8)$ & 0.006 \\
\hline Charlson comorbidity score & $1(0-1)$ & $1(0-2)$ & 0.024 & $1(0-2)$ & $1(0-2)$ & 0.022 \\
\hline \multicolumn{7}{|l|}{ Procedure } \\
\hline Pneumonectomy & $22(15)$ & $16(9)$ & 0.302 & $10(11)$ & $11(12)$ & 0.087 \\
\hline Bilobectomy & $10(7)$ & $9(5)$ & & $5(5)$ & $6(7)$ & \\
\hline Lobectomy & $106(75)$ & $160(85)$ & & $74(80)$ & $73(79)$ & \\
\hline Segmentectomy & $4(3)$ & $2(1)$ & & $3(3)$ & $2(2)$ & \\
\hline Wedge & $0(0)$ & $1(0.5)$ & & $0(0)$ & $0(0)$ & \\
\hline Laterality & & & 0.062 & & & 0.044 \\
\hline Right & $78(55)$ & $109(58)$ & & $52(57)$ & $54(59)$ & \\
\hline Left & $64(45)$ & $79(42)$ & & $40(43)$ & $38(41)$ & \\
\hline \multicolumn{7}{|l|}{ Histologic subtype } \\
\hline Adenocarcinoma & $69(49)$ & $109(58)$ & 0.189 & $45(49)$ & $44(48)$ & 0.077 \\
\hline Squamous & $43(30)$ & $47(25)$ & & $27(29)$ & $30(33)$ & \\
\hline Large cell, mixed, other & $30(21)$ & $32(17)$ & & $20(22)$ & $18(20)$ & \\
\hline \multicolumn{7}{|l|}{ Clinical stage } \\
\hline IB & $11(8)$ & $59(31)$ & 0.8 & $9(10)$ & $11(12)$ & 0.078 \\
\hline IIA & $20(14)$ & $44(23)$ & & $14(15)$ & $14(15)$ & \\
\hline IIB & $60(42)$ & $58(31)$ & & $42(46)$ & $42(46)$ & \\
\hline IIIA & $51(36)$ & $27(14)$ & & $27(29)$ & $25(27)$ & \\
\hline \multicolumn{7}{|l|}{ Clinical nodal stage } \\
\hline 0 & $86(61)$ & $152(81)$ & 0.457 & $55(60)$ & $59(64)$ & 0.09 \\
\hline 1 & $56(39)$ & $36(19)$ & & $37(40)$ & $33(36)$ & \\
\hline \multicolumn{7}{|l|}{ Clinical $\mathrm{T}$ stage } \\
\hline $2 \mathrm{a}$ & $25(18)$ & $71(38)$ & 0.583 & $22(24)$ & $21(23)$ & 0.072 \\
\hline $2 b$ & $32(23)$ & $53(28)$ & & $21(23)$ & $22(24)$ & \\
\hline 3 & $57(40)$ & $48(26)$ & & $33(36)$ & $35(38)$ & \\
\hline 4 & $28(20)$ & $16(9)$ & & $16(17)$ & $14(15)$ & \\
\hline Preinduction tumor $\mathrm{SUV}_{\max }$ & $13.8(10.9-18.0)$ & $11(7.4-15.0)$ & 0.526 & $12.7(9.2-17.3)$ & $12.8(9.0-16.4)$ & 0.081 \\
\hline
\end{tabular}

Data are no. (\%) or median (interquartile range), unless otherwise noted. An ASMD less than 0.1 indicates balance in the covariate between the 2 groups. ASMD, Absolute standardized mean difference; $B M I$, body mass index; $S U V_{\max }$, maximum standardized uptake value.

RECIST response and pathologic response, are listed in Tables E3, E4, and E5. We found that RECIST response was significantly associated with DFS $(P=.035)$ but not OS $(P=.13)$ (Figure 3, $A$ and $B)$. Kaplan-Meier curves for MPR versus no MPR indicated better OS and DFS among patients with an MPR to NC (Figure 3, $C$ and $D$ ). Because MPR status was available for a subset of patients in the NC group (92/142), we did not perform a log-rank test in this subanalysis (Figure 3,C and $D$ ). Fourteen patients in our study (15\%) had an MPR to NC: $5(36 \%)$ classified as stable disease and $9(64 \%)$ classified as partial response by RECIST. We also found that ypStage was associated with DFS $(P<.001)$ but not OS $(P=.1)$ (Figure 3, $E$ and $F$ ).

\section{DISCUSSION}

Our primary observation is that timing of chemotherapy (whether before or after surgery) was not associated with 


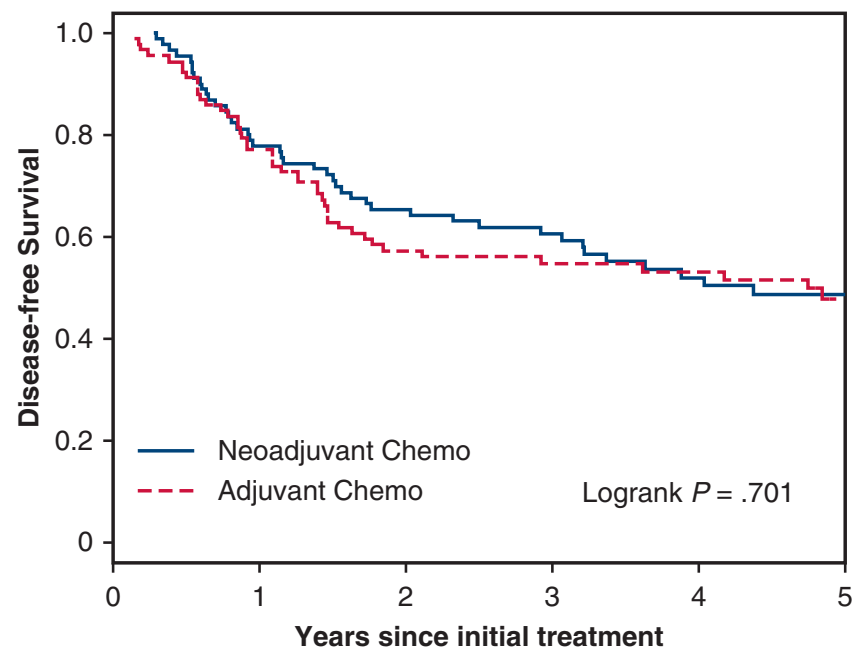

No. At Risk

Neoadjuvant Chemo 92

Adjuvant Chemo 92

DFS (\%)

Neoadjuvant Chemo 100

Adjuvant Chemo 100

$95 \% \mathrm{Cl}$

Neoadjuvant Chemo

Adjuvant Chemo

A

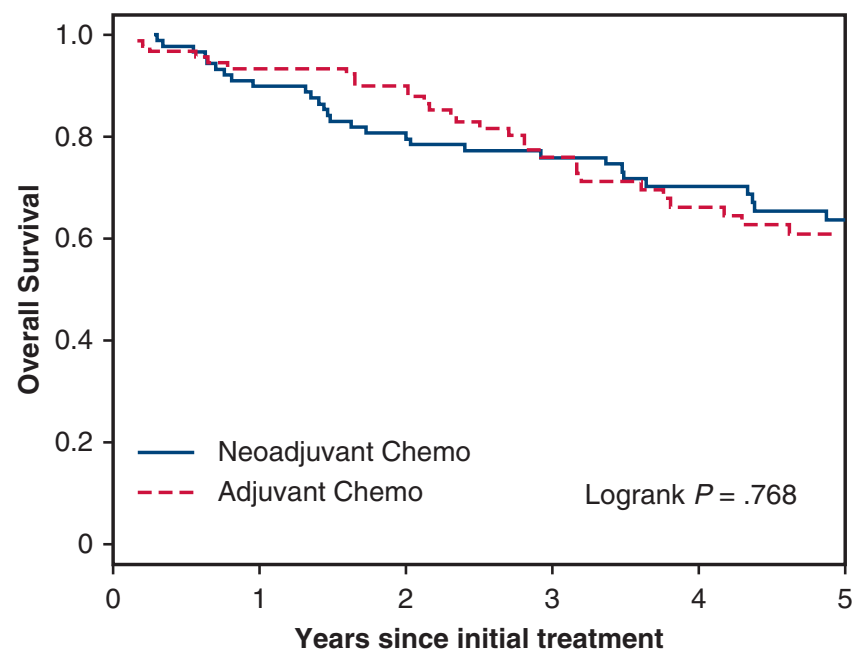

No. At Risk

Neoadjuvant Chemo 92

Adjuvant Chemo 92

os (\%)

Neoadjuvant Chemo 100

Adjuvant Chemo 100

$95 \% \mathrm{Cl}$

Neoadjuvant Chemo

Adjuvant Chemo

$\begin{array}{llllll}- & (70,87) & (56,76) & (51,72) & (42,64) & (39,61) \\ - & (69,86) & (48,68) & (45,66) & (44,65) & (38,60)\end{array}$

69

71

78

77

$(69,86)$

$(48,68)$

$(45,66)$

$(44,65)$

$(38,60)$

B

FIGURE 2. Five-year DFS (A) and OS (B) for the neoadjuvant versus adjuvant group using the propensity-matched cohort. Chemo, Chemotherapy; $D F S$, disease-free survival; $C I$, confidence interval; $O S$, overall survival. 
TABLE 2. Multivariable Cox proportional hazards model for disease-free and overall survival

\begin{tabular}{|c|c|c|c|c|}
\hline \multirow[b]{2}{*}{ Variable } & \multicolumn{2}{|c|}{ Disease-free survival } & \multicolumn{2}{|c|}{ Overall survival } \\
\hline & HR $(95 \%$ CI $)$ & $P$ & HR $(95 \%$ CI $)$ & $P$ \\
\hline Chemotherapy (vs neoadjuvant) & 1.00 & - & 1.00 & - \\
\hline Adjuvant & $1.10(0.64-1.90)$ & .737 & $1.21(0.63-2.30)$ & .572 \\
\hline Age & $1.00(0.96-1.04)$ & .805 & $1.01(0.96-1.06)$ & .765 \\
\hline Gender & - & - & 1.00 & - \\
\hline Male & - & - & $2.03(0.58-7.11)$ & .270 \\
\hline Procedure (vs pneumonectomy) & 1.00 & & 1.00 & - \\
\hline Lobectomy, bilobectomy, segmentectomy & $0.58(0.14-2.45)$ & .461 & $0.54(0.10-2.89)$ & .467 \\
\hline Histologic subtype (vs adenocarcinoma) & 1.00 & - & 1.00 & - \\
\hline Squamous & $0.48(0.17-1.39)$ & .175 & $0.68(0.20-2.34)$ & .54 \\
\hline Large cell, mixed, other & $0.62(0.22-1.75)$ & .367 & $1.13(0.33-3.87)$ & .84 \\
\hline Clinical stage (vs IB) & 1.00 & - & 1.00 & - \\
\hline IIA & $0.77(0.15-4.06)$ & .759 & $1.34(0.16-10.96)$ & .787 \\
\hline IIB & $3.30(0.59-18.5)$ & .175 & $2.74(0.35-21.71)$ & .339 \\
\hline IIIA & $2.86(0.45-18.1)$ & .264 & $1.72(0.18-16.23)$ & .635 \\
\hline
\end{tabular}

$H R$, Hazard ratio; $C I$, confidence interval.

DFS or OS in patients with clinically higher T-stage (T2-4), N0-1 NSCLC. At present, there are more robust data on AC than NC for the treatment of patients with cT2-4N0-1M0 NSCLC. In 2008, Pignon and colleagues ${ }^{4}$ reported that $\mathrm{AC}$ is associated with an $11 \%$ decrease in risk of death, a $5.8 \%$ benefit in DFS, and a $5.4 \%$ absolute improvement in OS, compared with surgery alone. Likewise, the superiority of NC plus surgery over surgery alone for patients with cT2-4N0-1M0 NSCLC has been established by other trials. SWOG S9900 evaluated neoadjuvant carboplatinpaclitaxel in patients with stage IB-IIIA NSCLC (excluding patients with Pancoast tumors and cN2 disease). ${ }^{17}$ This trial, which was powered to detect a $33 \%$ increase in median survival, was closed early after trials of AC establishing its efficacy were published. However, analysis of the abbreviated SWOG 9900 trial revealed a 9\% improvement in both progression-free survival and OS for NC plus surgery versus surgery alone. ${ }^{17}$ Another trial, which similarly closed early and evaluated neoadjuvant cisplatin and gemcitabine, found an $8 \%$ improvement in 3-year OS for NC versus surgery alone. ${ }^{18}$ Similar to our study, Lim and colleagues ${ }^{19}$ reported a meta-analysis of 32 clinical trials and found no difference in OS or DFS between NC or AC in

TABLE 3. Chemotherapy tolerance between the neoadjuvant and adjuvant groups

\begin{tabular}{lccc}
\hline \multicolumn{1}{c}{ Category } & $\begin{array}{c}\text { Neoadjuvant } \\
(\mathbf{N}=\mathbf{9 2} ; \mathbf{5 0} \%)\end{array}$ & $\begin{array}{c}\text { Adjuvant } \\
(\mathbf{N}=\mathbf{9 2} ; \mathbf{5 0} \%)\end{array}$ & $\boldsymbol{P}$ \\
\hline Full dose of chemotherapy & $72(78)$ & $58(63)$ & .014 \\
Full cycles of chemotherapy & $84(91)$ & $72(78)$ & .005 \\
$\begin{array}{l}\text { Grade } \geq 3 \text { adverse reaction to } \\
\text { chemotherapy }\end{array}$ & $14(15)$ & $35(38)$ & .001 \\
\hline
\end{tabular}

patients with resected NSCLC. In contrast, a 2014 metaanalysis comprising 15 randomized trials $(n=2385$ patients) compared NC followed by surgery and surgery alone and found that $\mathrm{NC}$ was associated with better recurrencefree survival and distant recurrence rates and a $5 \%$ absolute improvement in OS, with an HR identical to that of the pooled adjuvant studies. ${ }^{20}$ Collectively, the available evidence suggests that $\mathrm{NC}$ may offer a survival advantage over surgery alone for patients with locoregionally advanced NSCLC.

In contrast to the above studies, our study directly compares $\mathrm{NC}$ with $\mathrm{AC}$ - the current standard of care-in patients with locoregionally advanced (non-cN2) NSCLC. As discussed, multiple studies have demonstrated that $\mathrm{NC}$ and $\mathrm{AC}$ are associated with a survival benefit over surgery alone; however, few studies have directly compared the 2 chemotherapy approaches. ${ }^{4,17,18}$ Only the NATCH trial evaluated both $\mathrm{NC}$ versus surgery alone and $\mathrm{AC}$ versus surgery alone in patients with T1N0-T3N1 NSCLC. In that study, the addition of chemotherapy (NC or AC) to surgery had no effect on DFS; however, the study included an overly optimistic power analysis of a $15 \%$ difference in DFS at 5 years and a preponderance of patients with clinical stage I disease, for whom chemotherapy has been shown to have limited benefit. ${ }^{7,20-23}$ Although our findings are similar to those from the NATCH trial and the meta-analysis comparing $\mathrm{NC}$ followed by surgery and surgery alone, there are important differences. ${ }^{20,24}$ In the NATCH study and the meta-analysis, $75 \%$ and $50 \%$ of the NC cohorts, respectively, had clinical stage I disease. ${ }^{7,17}$ In contrast, our propensity score-matched cohort consisted of patients with higher-stage tumors $(53 \% \mathrm{cT} 3-4,38 \% \mathrm{cN} 1)$, and these patients are more likely to benefit from the addition of chemotherapy to their treatment plan. In addition, in 


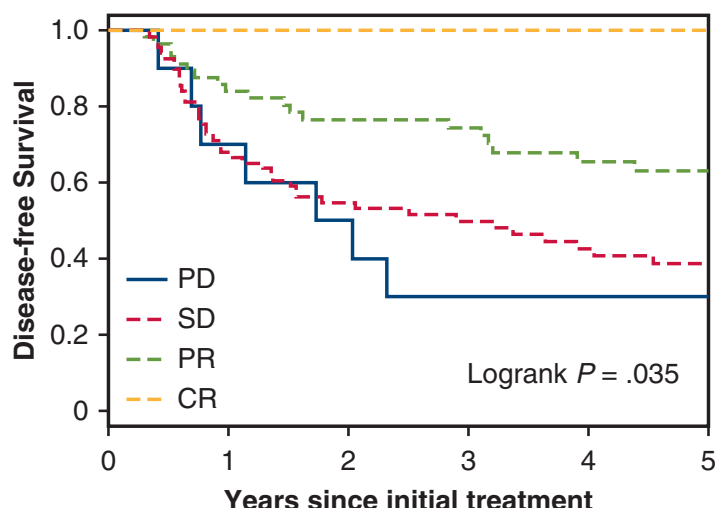

No. At Risk

$\begin{array}{lllllll}\text { PD } & 10 & 7 & 5 & 2 & 1 & 1\end{array}$

SD 70

PR 57

CR 1

DFS (\%)

PD 100

SD 100

PR 100

CR 100

$95 \% \mathrm{Cl}$

$\begin{array}{llcllll}\mathrm{PD} & - & (47,100) & (27,93) & (12,77) & (12,77) & (12,77) \\ \mathrm{SD} & - & (58,80) & (44,68) & (39,63) & (32,57) & (28,53) \\ \mathrm{PR} & - & (75,94) & (66,89) & (64,87) & (54,80) & (51,78)\end{array}$

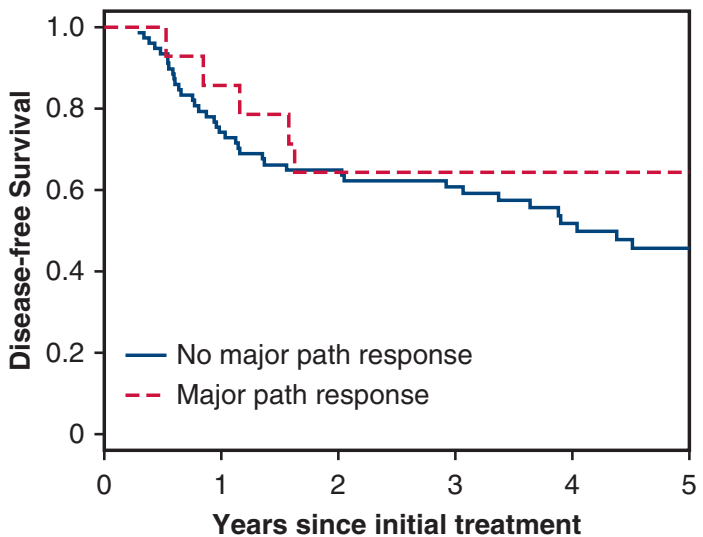

No. At Risk

$\begin{array}{lllllll}\text { No MPR } & 78 & 57 & 49 & 38 & 27 & 19\end{array}$ $\begin{array}{lllllll}\text { MPR } & 14 & 12 & 9 & 7 & 6 & 4\end{array}$

DFS (\%)

$\begin{array}{lllllll}\text { No MPR } & 100 & 74 & 65 & 61 & 52 & 46\end{array}$

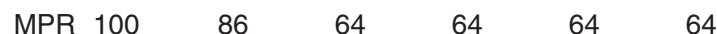

$95 \% \mathrm{Cl}$

No MPR - $\quad(65,85) \quad(55,77) \quad(51,73) \quad(41,65) \quad(35,60)$

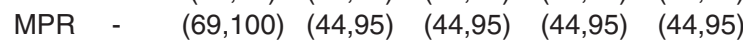

C

FIGURE 3. Five-year DFS and OS by RECIST response (A, B), MPR (C, D), and ypStage (E, F). $P D$, Progressive disease; $S D$, stable disease; $P R$, partial response; $C R$, complete response; $D F S$, disease-free survival; $C I$, confidence interval; $O S$, overall survival; path, pathologic; $M P R$, major pathologic response.

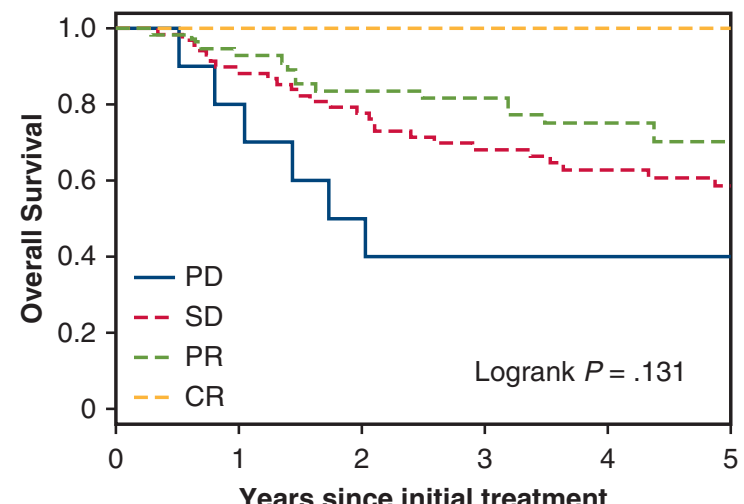

No. At Risk

$\begin{array}{ccccccc}\text { PD } & 10 & 8 & 5 & 3 & 2 & 2 \\ \text { SD } & 70 & 59 & 51 & 40 & 34 & 25 \\ \text { PR } 57 & 51 & 45 & 39 & 32 & 26 \\ \text { CR } 1 & 1 & 1 & 1 & 1 & 1 \\ \text { OS (\%) } & & & & & \\ \text { PD 100 } & 80 & 50 & 40 & 40 & 40 \\ \text { SD 100 } & 88 & 78 & 68 & 63 & 58 \\ \text { PR 100 } & 93 & 84 & 82 & 75 & 70 \\ \text { CR 100 } & 100 & 100 & 100 & 100 & 100 \\ 95 \% \text { CI } & & & & & \\ \text { PD - } & (59,100) & (27,93) & (19,85) & (19,85) & (19,85) \\ \text { SD - } & (81,96) & (68,88) & (58,80) & (52,76) & (47,72) \\ \text { PR - } & (86,100) & (74,94) & (72,93) & (64,88) & (59,84) \\ \text { CR - } & - & - & - & - & -\end{array}$

B

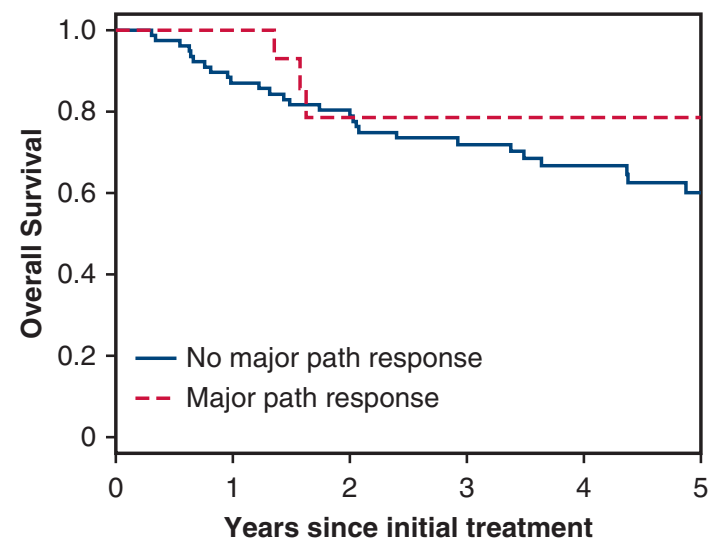

No. At Risk

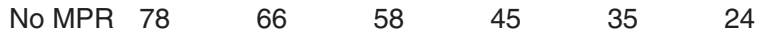

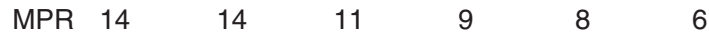

OS (\%)

$\begin{array}{llllll}\text { No MPR } 100 & 87 & 79 & 72 & 67 & 60 \\ \text { MPR } 100 & 100 & 79 & 79 & 79 & 79\end{array}$

$95 \% \mathrm{Cl}$

No MPR - $\quad(80,95) \quad(70,89) \quad(62,83) \quad(56,79) \quad(49,74)$

MPR - $(100,100)(60,100)(60,100)(60,100)(60,100)$ 


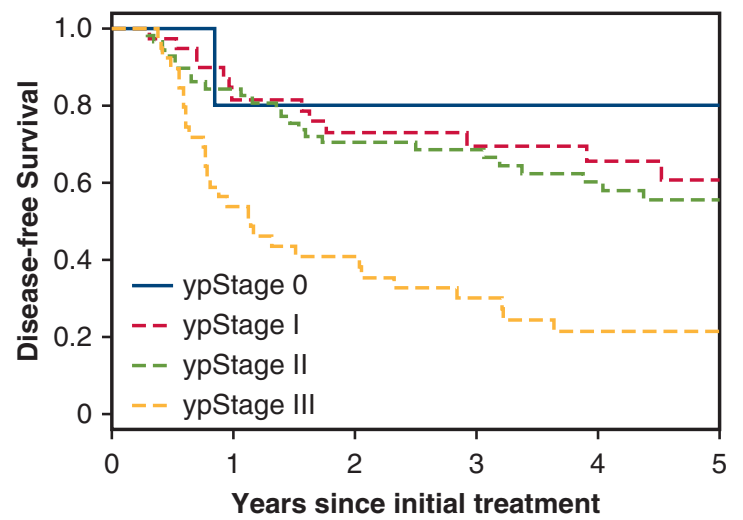

No. At Risk ypStage $0 \quad 5$ ypStage I 39 ypStage II 59 ypStage III 39

DFS (\%) ypStage 0100 ypStage I 100 ypStage II 100 ypStage III 100 $95 \% \mathrm{Cl}$ ypStage 0 ypStage I ypStage II ypStage III -

E

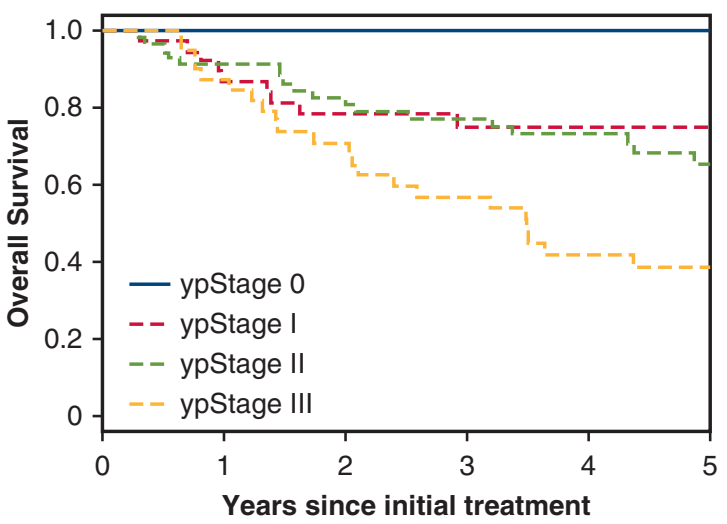

No. At Risk ypStage $0 \quad 5$ ypStage I 39 ypStage II 59 ypStage III 39

DFS (\%) ypStage 0100 ypStage I 100 ypStage II 100

ypStage III 100

$95 \% \mathrm{Cl}$

$\begin{array}{ccccc}5 & 5 & 4 & 4 & 4 \\ 32 & 28 & 22 & 19 & 17 \\ 52 & 46 & 40 & 33 & 22 \\ 33 & 26 & 20 & 14 & 12\end{array}$

$\begin{array}{lllll}100 & 100 & 100 & 100 & 100\end{array}$

$\begin{array}{lllll}87 & 78 & 75 & 75 & 75\end{array}$

$\begin{array}{lllll}91 & 81 & 77 & 73 & 65\end{array}$

$\begin{array}{lllll}87 & 71 & 57 & 42 & 39\end{array}$

$\begin{array}{ccccccc}(52,100) & (52,100) & (52,100) & (52,100) & (52,100) & \text { ypStage 0 } & \text { - } \\ (70,95) & (60,89) & (56,87) & (51,84) & (46,81) & \text { ypStage I } & - \\ (76,94) & (59,83) & (58,82) & (49,75) & (44,71) & \text { ypStage II } & - \\ (40,72) & (28,60) & (19,49) & (12,40) & (12,40) & \text { ypStage III } & \text { - }\end{array}$

$(77,98) \quad(66,93)$

$(84,99) \quad(71,92)$

$(62,91) \quad(62,91)$

$(67,89) \quad(63,86)$

$(62,91)$

$(77,98) \quad(58,87)$

$(43,75)$

$(28,62)$

$(53,80)$

$(25,59)$

FIGURE 3. Continued.

the NATCH trial, all patients received carboplatin-based chemotherapy, whereas in the present study $63 \%$ of patients received cisplatin-based chemotherapy; however, it should be noted that the meta-analysis found no difference in survival between these 2 regimens. ${ }^{17}$ More patients in the present study had adenocarcinoma histologic subtype (48\% vs $29 \%$ in NATCH). Moreover, in both NATCH and the metaanalysis, women made up a small portion of the study population $(13 \%$ in NATCH and $22 \%$ in the meta-analysis vs $43 \%$ in the present study). ${ }^{7,20}$ Therefore, our study population perhaps more closely reflects the population of patients with NSCLC who would receive either NC or $\mathrm{AC}$, and is likely better suited to evaluate the putative benefits of NC.

In our study, significantly more patients in the NC group received the full dose and full cycle of chemotherapy than in the AC group. In addition, there were fewer high-grade adverse events in the $\mathrm{NC}$ group. Our findings support those of the NATCH study ${ }^{7}$ in that NC is associated with better chemotherapy tolerance than AC. Unfortunately, in both studies, the improved tolerance among patients receiving $\mathrm{NC}$ was not associated with improved DFS or OS, although a similar improvement in compliance has been shown to be associated with improved survival in breast cancer. ${ }^{25}$ Despite the lack of a survival benefit, the other benefits of $\mathrm{NC}$, especially the improved tolerance and the ability to monitor response and stop or alter therapy if no response is observed, can make it appealing for patients and clinicians.

Our findings that RECIST response to NC was associated with improved DFS but not OS are similar to those from a study from MD Anderson that evaluated OS in 160 patients with clinical stage I to IV NSCLC who underwent NC followed by resection. Although the authors of that study initially found that RECIST response was associated with OS, when pathologic response was included in multivariable analysis, the association was no longer present. ${ }^{26}$ In contrast, a group from Moffitt Cancer Center evaluated RECIST in 89 patients and found that CT RECIST response, but not PET RECIST response was associated with OS and that the association was more pronounced among patients with higher-stage disease. ${ }^{27}$ It is possible that the lack of association between OS and RECIST response in our study can be explained by our exclusion of patients with $\mathrm{cN} 2$ or higher stage disease. The aforementioned studies had more patients with stage III/IV disease 
(56\% in the MD Anderson study and 40\% in the Moffitt Cancer Center study). In addition, both studies divided the RECIST response into 2 groups (partial response/ complete response vs progressive disease/stable disease); we chose to examine the criteria as 4 distinct groups.

It has been suggested that MPR is an assessable and reliable surrogate measurement of survival after $\mathrm{NC}$ for NSCLC. ${ }^{11}$ MPR rates after NC were not reported in the NATCH or CHEST trials or in the meta-analysis. ${ }^{7,18,20}$ In our study, the MPR rate after NC was $15 \%$, which is similar to that in other studies ${ }^{26,28}$ but significantly less than the $43 \%$ recently reported in a small series of patients with NSCLC receiving 2 cycles of neoadjuvant nivolumab followed by surgical resection. ${ }^{8}$ Unfortunately, there is a significant discordance between RECIST response (CT and PET) and MPR in patients with NSCLC treated with NC. William and colleagues ${ }^{26}$ found a $41 \%$ discordance rate between CT RECIST response and histopathologic response; we observed a discordance rate of at least $36 \%$ in our study.

\section{Study Limitations}

The limitations of our study include its retrospective, single-institution nature, which may limit the generalizability of the results. Additionally, we used clinical stage to match cohorts, which has known inaccuracies, but has been used by other groups when evaluating outcomes after NC. ${ }^{17}$ Some patients may develop progressive disease during $\mathrm{NC}$ and are unable to undergo surgery; although this number is likely to be small $(3 \%-4 \%$ in published trials of NC), we cannot account for these patients in our data set. ${ }^{17,18}$ Although in our unmatched cohort, more patients had an incomplete resection in the NC group, the Lancet meta-analysis found that preoperative chemotherapy was not associated with ability to undergo complete resection (odds ratio, 0.88), and our rate of $\mathrm{R} 2$ resection in the $\mathrm{NC}$ group $(6.2 \%)$ is comparable to that published in the previous trials. ${ }^{7}$ We also do not know the number of patients who underwent surgery and were eligible for AC but did not receive it. These are common limitations of a retrospective design that would be addressed in a prospective intentionto-treat study. Finally, although this was a large study, it still may be underpowered to fully address the effect of the timing of chemotherapy on DFS or OS in patients with advanced, non-N2 NSCLC.

\section{CONCLUSIONS}

Despite the higher levels of tolerance and full doses and full cycles of chemotherapy in the NC group, neoadjuvant administration did not result in better DFS or OS, compared with adjuvant administration, among patients with cT2-4N0-1M0 NSCLC who had R0 resection. However, RECIST response to chemotherapy was associated with better DFS. Our study provides a contemporary benchmark against which to compare outcomes of future neoadjuvant regimens, including immunotherapy, for locally advanced NSCLC. It also amplifies the need to more fully understand and develop genomic, molecular, or pathologic biomarkers (alone or in combination) to more accurately predict which tumors will respond to neoadjuvant or adjuvant regimens as part of the treatment paradigm for patients with surgically resectable NSCLC.

\section{Conflict of Interest Statement}

J.E.C. has received consulting fees from AstraZeneca, Merck, Genentech, and BMS and research funding from AstraZeneca, Genentech, and Bristol-Myers Squibb. M.G.K. has received consulting fees from AstraZeneca, Pfizer, and Regeneron. W.D.T. has served in a nonpaid consulting role for Genentech. All other authors have nothing to disclose with regard to commercial support.

\section{References}

1. Brandt WS, Bouabdallah I, Tan KS, Park BJ, Adusumilli PS, Molena D, et al. Factors associated with distant recurrence following R0 lobectomy for pN0 lung adenocarcinoma. J Thorac Cardiovasc Surg. 2018;155:1212-24.e1213.

2. National Comprehensive Cancer Network. Clinical Practice Guidelines in Oncology (NCCN guidelines): Non-Small Cell Lung Cancer. Version 6. August 17, 2018. Available at: https://www.nccn.org/professionals/physician_gls/ default.aspx. Accessed August 18, 2018.

3. Berry MF, Coleman BK, Curtis LH, Worni M, D'Amico TA, Akushevich I. Benefit of adjuvant chemotherapy after resection of stage II (T1-2N1M0) nonsmall cell lung cancer in elderly patients. Ann Surg Oncol. 2015;22:642-8.

4. Pignon JP, Tribodet H, Scagliotti GV, Douillard JY, Shepherd FA, Stephens RJ, et al. Lung adjuvant cisplatin evaluation: a pooled analysis by the LACE collaborative group. J Clin Oncol. 2008;26:3552-9.

5. Arriagada R, Bergman B, Dunant A, Le Chevalier T, Pignon JP, Vansteenkiste J, International Adjuvant Lung Cancer Trial Collaborative Group. Cisplatin-based adjuvant chemotherapy in patients with completely resected non-small-cell lung cancer. N Engl J Med. 2004;350:351-60.

6. Chaft JE, Dunphy M, Naidoo J, Travis WD, Hellmann M, Woo K, et al. Adaptive neoadjuvant chemotherapy guided by (18)F-FDG PET in resectable non-small cell lung cancers: the NEOSCAN trial. J Thorac Oncol. 2016;11:537-44.

7. Felip E, Rosell R, Maestre JA, Rodríguez-Paniagua JM, Morán T, Astudillo J, et al. Preoperative chemotherapy plus surgery versus surgery plus adjuvant chemotherapy versus surgery alone in early-stage non-small-cell lung cancer. $J$ Clin Oncol. 2010;28:3138-45.

8. Forde PM, Chaft JE, Smith KN, Anagnostou V, Cottrell TR, Hellmann MD, et al. Neoadjuvant PD-1 blockade in resectable lung cancer. N Engl J Med. 2018;378: 1976-86.

9. Ripley RT, Suzuki K, Tan KS, Adusumilli PS, Huang J, Park BJ, et al. Postinduction positron emission tomography assessment of $\mathrm{N} 2$ nodes is not associated with ypN2 disease or overall survival in stage IIIA non-small cell lung cancer. $J$ Thorac Cardiovasc Surg. 2016;151:969-77. 979 e961-963.

10. Eisenhauer EA, Therasse P, Bogaerts J, Schwartz LH, Sargent D, Ford R, et al. New response evaluation criteria in solid tumours: revised RECIST guideline (version 1.1). Eur J Cancer. 2009;45:228-47.

11. Hellmann MD, Chaft JE, William WN, Rusch V, Pisters KM, Kalhor N, et al. Pathological response after neoadjuvant chemotherapy in resectable non-smallcell lung cancers: proposal for the use of major pathological response as a surrogate endpoint. Lancet Oncol. 2014;15:e42-50.

12. Attaar A, Winger DG, Luketich JD, Schuchert MJ, Sarkaria IS, Christie NA, et al. A clinical prediction model for prolonged air leak after pulmonary resection. $J$ Thorac Cardiovasc Surg. 2017;153:690-9.e692.

13. Dugan KC, Laxmanan B, Murgu S, Hogarth DK. Management of persistent air leaks. Chest. 2017; 152:417-23.

14. Martini N, Melamed MR. Multiple primary lung cancers. J Thorac Cardiovasc Surg. 1975;70:606-12. 
15. Austin PC. Optimal caliper widths for propensity-score matching when estimating differences in means and differences in proportions in observational studies. Pharm Stat. 2011;10:150-61.

16. Austin PC. An introduction to propensity score methods for reducing the effects of confounding in observational studies. Multivariate Behav Res. 2011;46: 399-424.

17. Pisters KM, Vallieres E, Crowley JJ, Franklin WA, Bunn PA Jr, Ginsberg RJ, et al. Surgery with or without preoperative paclitaxel and carboplatin in early-stage non-small-cell lung cancer: Southwest oncology group trial S9900, an intergroup, randomized, phase III trial. J Clin Oncol. 2010;28:1843-9.

18. Scagliotti GV, Pastorino U, Vansteenkiste JF, Spaggiari L, Facciolo F, Orlowski TM, et al. Randomized phase III study of surgery alone or surgery plus preoperative cisplatin and gemcitabine in stages IB to IIIA non-small-cell lung cancer. J Clin Oncol. 2012;30:172-8.

19. Lim E, Harris G, Patel A, Adachi I, Edmonds L, Song F. Preoperative versus postoperative chemotherapy in patients with resectable non-small cell lung cancer: systematic review and indirect comparison meta-analysis of randomized trials. J Thorac Oncol. 2009; 4:1380-8.

20. Preoperative chemotherapy for non-small-cell lung cancer: a systematic review and meta-analysis of individual participant data. Lancet. 2014;383:1561-71.

21. Winton T, Livingston R, Johnson D, Rigas J, Johnston M, Butts C, et al. Vinorelbine plus cisplatin vs. observation in resected non-small-cell lung cancer. $N$ Engl J Med. 2005;352:2589-97.

22. Strauss GM, Herndon JE II, Maddaus MA, Johnstone DW, Johnson EA, Harpole DH, et al. Adjuvant paclitaxel plus carboplatin compared with observation in stage IB non-small-cell lung cancer: CALGB 9633 with the cancer and leukemia group B, radiation therapy oncology group, and north central cancer treatment group study groups. J Clin Oncol. 2008;26:5043-51.
23. Douillard JY, Rosell R, De Lena M, Carpagnano F, Ramlau R, GonzálesLarriba JL, et al. Adjuvant vinorelbine plus cisplatin versus observation in patients with completely resected stage IB-IIIA non-small-cell lung cancer (Adjuvant Navelbine International Trialist Association [ANITA]): a randomised controlled trial. Lancet Oncol. 2006;7:719-27.

24. McElnay P, Lim E. Adjuvant or neoadjuvant chemotherapy for NSCLC. J Thorac Dis. 2014;6(Suppl 2):S224-7.

25. Bonadonna G, Valagussa P, Moliterni A, Zambetti M, Brambilla C. Adjuvant cyclophosphamide, methotrexate, and fluorouracil in node-positive breast cancer: the results of 20 years of follow-up. N Engl J Med. 1995; 332:901-6.

26. William WN Jr, Pataer A, Kalhor N, Correa AM, Rice DC, Wistuba II, et al Computed tomography RECIST assessment of histopathologic response and prediction of survival in patients with resectable non-small-cell lung cancer after neoadjuvant chemotherapy. J Thorac Oncol. 2013;8:222-8.

27. Tanvetyanon T, Eikman EA, Sommers E, Robinson L, Boulware D, Bepler G Computed Tomography response, but not positron emission tomography scan response, predicts survival after neoadjuvant chemotherapy for resectable nonsmall-cell lung cancer. J Clin Oncol. 2008;26:4610-6.

28. Chaft JE, Rusch V, Ginsberg MS, Paik PK, Finley DJ, Kris MG, et al. Phase II trial of neoadjuvant bevacizumab plus chemotherapy and adjuvant bevacizumab in patients with resectable nonsquamous non-small-cell lung cancers. $J$ Thorac Oncol. 2013;8:1084-90.

Key Words: non-small cell lung cancer, neoadjuvant chemotherapy, adjuvant chemotherapy, propensitymatched analysis, survival 
APPENDIX TABLE 1. Chemotherapy regimens for propensity-matched cohort

\begin{tabular}{lcc}
\hline \multicolumn{1}{c}{ Chemotherapy regimen } & Neoadjuvant chemotherapy N (\%) & Adjuvant chemotherapy N $(\%)$ \\
\hline Carboplatin, docetaxel & $10(11)$ & $1(1.1)$ \\
\hline Cisplatin, gemcitabine & $14(15)$ & $1(1.1)$ \\
\hline Cisplatin, vinorelbine & $2(2.2)$ & $34(37)$ \\
Carboplatin, paclitaxel & $9(9.8)$ & $12(13)$ \\
Cisplatin, docetaxel, gemcitabine & $1(1.1)$ & $0(0)$ \\
Carboplatin, gemcitabine & $7(7.6)$ & $7(7.6)$ \\
Cisplatin, docetaxel & $22(24)$ & $6(6.5)$ \\
Cisplatin, etoposide & $3(3.3)$ & $3(3.3)$ \\
Cisplatin, pemetrexed & $17(18)$ & $9(9.8)$ \\
Carboplatin, pemetrexed & $7(7.6)$ & $14(15)$ \\
Cisplatin, vinorelbine then carboplatin, paclitaxel & $0(0)$ & $2(2.2)$ \\
Cisplatin, docetaxel, vinorelbine & $0(0)$ & $1(1.1)$ \\
Carboplatin, paclitaxel, vinorelbine & $0(0)$ & $1(1.1)$ \\
Carboplatin, paclitaxel, pemetrexed & $0(0)$ & $1(1.1)$ \\
\hline
\end{tabular}

TABLE E1. Clinicopathologic variables after propensity score matching

\begin{tabular}{|c|c|c|}
\hline Variable & Neoadjuvant $(\mathrm{N}=92 ; 50 \%)$ & Adjuvant $(\mathrm{N}=92 ; 50 \%)$ \\
\hline Forced expiratory volume in $1 \mathrm{~s}$ & $81.0(71.5-96.5)$ & $87.0(77.0-100.0)$ \\
\hline Diffusion lung carbon monoxide, $\%$ & $76.0(60.0-86.0)$ & $83.2(66.5-96.5)$ \\
\hline \multicolumn{3}{|c|}{ Pretreatment biopsy of $\mathrm{cN} 2$ nodes to exclude $\mathrm{cN} 2$ disease } \\
\hline No & $62(67)$ & $70(76)$ \\
\hline Yes & $30(33)$ & $22(24)$ \\
\hline Pathologic tumor size, $\mathrm{cm}$ & $3.8(2.8-5.4)$ & $5.4(4.5-6.5)$ \\
\hline \multicolumn{3}{|l|}{ Pathologic tumor stage } \\
\hline 0 & $4(4)$ & $0(0)$ \\
\hline 1 & $20(22)$ & $3(3)$ \\
\hline 2 & $32(35)$ & $29(32)$ \\
\hline 3 & $25(27)$ & $40(43)$ \\
\hline 4 & $11(12)$ & $20(22)$ \\
\hline \multicolumn{3}{|l|}{ Pathologic nodal stage } \\
\hline 0 & $61(66)$ & $42(46)$ \\
\hline 1 & $20(22)$ & $36(39)$ \\
\hline 2 & $11(12)$ & $14(15)$ \\
\hline \multicolumn{3}{|l|}{ Overall pathologic stage } \\
\hline 0 & $3(3)$ & $0(0)$ \\
\hline I & $27(29)$ & $2(2)$ \\
\hline II & $40(43)$ & $44(48)$ \\
\hline III & $22(24)$ & $46(50)$ \\
\hline Lymphovascular invasion present $(\mathrm{n}=179)$ & $50(57)$ & $62(68)$ \\
\hline Pleural invasion present & $20(22)$ & $35(38)$ \\
\hline Pathologic tumor response, $\%$ necrosis $(\mathrm{n}=66)$ & $40.0(20.0-70.0)$ & NA \\
\hline Postoperative radiotherapy & $10(11)$ & $4(4)$ \\
\hline Number of cycles of chemotherapy $(n=183)$ & $4(3-4)$ & $4(4-4)$ \\
\hline
\end{tabular}

Data are no. (\%) or median (interquartile range), unless otherwise noted. 
TABLE E2. Types of grade $\geq 3$ complications neoadjuvant chemotherapy versus adjuvant chemotherapy propensity scorematched cohort*

\begin{tabular}{lcc}
\hline & NC $(\mathbf{N}=\mathbf{1 3})$ & $\mathbf{A C}(\mathbf{N}=\mathbf{6})$ \\
\hline Pneumothorax/air leak & 1 & 1 \\
Wound infection & 1 & 0 \\
\hline Pulmonary embolus & 1 & 1 \\
Pulmonary infection or respiratory failure & 4 & 3 \\
Cardiac tamponade & 1 & 0 \\
Acute kidney injury & 1 & 0 \\
Cardiac arrest & 1 & 0 \\
Myocardial infarction & 1 & 1 \\
\hline Acute respiratory distress syndrome & 1 & 0 \\
Sepsis & 1 & 0 \\
\hline Hyperglycemia & 0 & 1 \\
Small bowel obstruction & 1 & 0 \\
\hline Neuropathy & 1 & 0 \\
Total complications & 15 & 7 \\
\hline
\end{tabular}

$N C$, Neoadjuvant chemotherapy; $A C$, adjuvant chemotherapy. ${ }^{*}$ In the NC group, 1 patient had both wound infection and pulmonary embolus, 1 patient had both small bowel obstruction and acute kidney injury, 1 patient had neuropathy and pneumothorax, and 1 patient had both sepsis and acute respiratory distress syndrome. In the $\mathrm{AC}$ group, 1 patient had both respiratory failure and pneumonia.
TABLE E3. Clinicopathologic characteristics of entire neoadjuvant chemotherapy cohort $(n=142)$

\begin{tabular}{|c|c|}
\hline & $\mathbf{N}(\%)$ or median (IQR) \\
\hline \multicolumn{2}{|l|}{ Clinical tumor stage (cT) } \\
\hline $2 \mathrm{a}$ & $25(18 \%)$ \\
\hline $2 b$ & $32(23 \%)$ \\
\hline 3 & $57(40 \%)$ \\
\hline 4 & $28(20 \%)$ \\
\hline \multicolumn{2}{|l|}{ Clinical nodal stage $(\mathrm{cN})$} \\
\hline 0 & $86(61 \%)$ \\
\hline 1 & $56(39 \%)$ \\
\hline \multicolumn{2}{|l|}{ Clinical stage } \\
\hline IB & $11(7.7 \%)$ \\
\hline IIA & $20(14 \%)$ \\
\hline IIB & $60(42 \%)$ \\
\hline IIIA & $51(36 \%)$ \\
\hline \multicolumn{2}{|l|}{ RECIST } \\
\hline Complete response & $1(0.7 \%)$ \\
\hline Partial response & $57(40 \%)$ \\
\hline Stable disease & $70(49 \%)$ \\
\hline Progressive disease & $10(7.0 \%)$ \\
\hline Unknown & $4(2.8 \%)$ \\
\hline Postinduction tumor size, $\mathrm{cm}$ & $3.9(2.9-5.3)$ \\
\hline Pathologic tumor size (cm) & $4.0(2.9,5.8)$ \\
\hline \multicolumn{2}{|l|}{$\begin{array}{l}\text { Pathologic tumor stage } \\
\text { (patient) }\end{array}$} \\
\hline 0 & $7(4.9 \%)$ \\
\hline 1 & $26(18 \%)$ \\
\hline $1 b$ & $1(0.7 \%)$ \\
\hline 2 & $47(33 \%)$ \\
\hline $2 b$ & $1(0.7 \%)$ \\
\hline 3 & $40(28 \%)$ \\
\hline 4 & $20(14 \%)$ \\
\hline \multicolumn{2}{|l|}{ Pathologic nodal stage $(\mathrm{pN})$} \\
\hline 0 & $96(68 \%)$ \\
\hline 1 & $31(22 \%)$ \\
\hline 2 & $15(11 \%)$ \\
\hline \multicolumn{2}{|l|}{ Pathologic stage } \\
\hline 0 & $5(3.5 \%)$ \\
\hline I & $39(27 \%)$ \\
\hline II & $59(42 \%)$ \\
\hline III & $39(27 \%)$ \\
\hline $\begin{array}{l}\text { Lymphovascular invasion } \\
\text { present }(\mathrm{N}=322)\end{array}$ & $75(56 \%)$ \\
\hline Pleura invasion present & $30(21 \%)$ \\
\hline Percent necrosis $(\mathrm{N}=92)$ & $40.0(20.0,70.0)$ \\
\hline
\end{tabular}

Data are no. (\%) or median (IQR), unless otherwise noted. IQR, Interquartile range; RECIST, Response Evaluation Criteria In Solid Tumors. 
TABLE E4. Clinical and pathologic tumor staging for the neoadjuvant group

\begin{tabular}{|c|c|c|c|c|c|}
\hline \multirow[b]{2}{*}{ Clinical tumor stage } & \multicolumn{5}{|c|}{ Pathologic tumor stage } \\
\hline & $\mathbf{0}$ & 1 & 2 & 3 & 4 \\
\hline $2 \mathrm{a}(\mathrm{n}=29)$ & $0(3.5)$ & $1(44.8)$ & $2(37.9)$ & $3(10.39)$ & $4(3.4)$ \\
\hline $2 \mathrm{~b}(\mathrm{n}=33)$ & $1(3.03)$ & $13(27.3)$ & $11(48.5)$ & $3(12.12)$ & $1(9.09)$ \\
\hline $3(n=64)$ & $1(3.13)$ & $9(6.25)$ & $16(35.9)$ & $4(40.63)$ & $3(14.1)$ \\
\hline $4(n=36)$ & $2(8.33)$ & $4(5.56)$ & $23(11.1)$ & $26(38.9)$ & $9(36.1)$ \\
\hline
\end{tabular}

TABLE E5. Clinical and pathologic nodal staging for the neoadjuvant group

\begin{tabular}{lccrr}
\hline & \multicolumn{4}{c}{ Pathologic nodal stage } \\
\cline { 2 - 5 } Clinical nodal stage & $\mathbf{0}$ & $\mathbf{1}$ & $\mathbf{2}$ & \multicolumn{1}{c}{$\mathbf{X}$} \\
\hline 0 & $66(68.0)$ & $23(23.7)$ & $7(7.22)$ & $1(1.03)$ \\
1 & $37(39.8)$ & $17(18.3)$ & $10(10.8)$ & $1(1.08)$ \\
\hline
\end{tabular}

